BMJ Open

Diabetes

Research

\& Care

\title{
Stair ascending-descending exercise accelerates the decrease in postprandial hyperglycemia more efficiently than bicycle exercise
}

\author{
Tetsuo Takaishi, ${ }^{1}$ Tatsuya Hayashi ${ }^{2}$
}

To cite: Takaishi T, Hayashi T. Stair ascending-descending exercise accelerates the decrease in postprandial hyperglycemia more efficiently than bicycle exercise. BMJ Open Diab Res Care 2017;5:e000428. doi:10.1136/ bmjdrc-2017-000428

Received 5 April 2017 Revised 10 August 2017 Accepted 29 August 2017

\section{CrossMark}

${ }^{1}$ Graduate School of Natural Sciences, Nagoya City University, Nagoya, Japan ${ }^{2}$ Graduate School of Human and Environmental Studies, Kyoto University, Kyoto, Japan

Correspondence to Professor Tetsuo Takaishi; takaishi@nsc.nagoya-cu.ac.jp

\section{ABSTRACT}

Objective Stair climbing-descending exercise (ST-EX) is a convenient method to increase exercise intensity. We compared the acute effect of ST-EX on lowering postprandial hyperglycemia with that of constant bicycle exercise (BI-EX) performed at the same heart rate (HR). Research design and methods Seven people with type 2 diabetes and seven with impaired glucose tolerance volunteered for this study. The step rate for ST-EX and work rate for BI-EX were individually determined to correspond to high-moderate to low-vigorous intensity (HR $\sim 130$ beats per minute). For the ST-EX trial, the subjects performed 16 repetitions of walking down one flight of stairs followed by climbing up to the starting point ( 8 min in duration) 90 min after consuming a test meal. For the BI-EX trial, the subjects performed a constant pedaling exercise for the same duration at the same time after the meal.

Results The reduction in blood glucose (BG) level between 90 and 105 min after a meal was significantly greater for ST-EX $(-4.0 \pm 0.7 \mathrm{mmol} / \mathrm{L})$ than for BI-EX $(-2.7 \pm 0.9 \mathrm{mmol} / \mathrm{L})$. The net reduction in $B G$ between 90 and 105 min was also significantly greater for ST-EX $(-3.2 \pm 0.7 \mathrm{mmol} / \mathrm{L})$ than for BI-EX (-2.0 $\pm 0.6 \mathrm{mmol} / \mathrm{L})$. Serum insulin levels did not differ between the groups. Oxygen consumption for ST-EX was higher than that for BI-EX, but the blood lactate level and respiratory exchange ratio (RER) for ST-EX were lower than those for BI-EX.

Conclusions Compared with BI-EX performed at the same HR, ST-EX more rapidly decreased postprandial BG level with lower blood lactate and RER responses. A short bout of ST-EX may be clinically useful to acutely ameliorate $B G$ levels after meals.

\section{INTRODUCTION}

Acute aerobic exercise (AE) is an important modality to control blood glucose (BG) level in people with type 2 diabetes. AE should be initiated at a moderate intensity corresponding to $40 \%-60 \%$ of maximal aerobic capacity, but people with type 2 diabetes already exercising at a moderate intensity have been recommended to undertake $\mathrm{AE}$ at a higher intensity to obtain additional BG benefits. 1

\section{Significance of this study}

What is already known about this subject? Stair climbing-descending exercise (ST-EX) is an easyto perform method to increase exercise intensity in daily life.

What are the new findings?

ST-EX performed after a meal more rapidly decreased postprandial blood glucose level than bicycle exercise performed at the same heart rate.

How might these results change the focus of research or clinical practice?

ST-EX might be a clinically useful modality for efficient amelioration of postprandial hyperglycemia.

Postprandial hyperglycemia is an important risk factor for cardiovascular disease. ${ }^{2-4}$ The American Diabetes Association ${ }^{5}$ recommends that postprandial BG should be controlled to $<10.0 \mathrm{mmol} / \mathrm{L}$, particularly when hemoglobin A1c $(\mathrm{HbA1c})$ remains $>7.0 \%$ despite preprandial BG being well controlled to 4.4-7.2 mmol/L. However, postprandial hyperglycemia is prevalent in people with type 2 diabetes, including those with apparently good HbA1c levels. ${ }^{6}$

It has been shown that high-intensity AE effectively reduces postprandial BG level. ${ }^{7-10}$ However, it is notable that the reported protocols of high-intensity AE (eg, 6 bouts of $1 \mathrm{~min}$ high-intensity incline walking, ${ }^{9} 10$ bouts of 60 s high-intensity cycling, ${ }^{8}$ repeated cycles of 3 min of slow and fast walking for 1 hour, ${ }^{10}$ and 4 bouts of high-intensity cycling for a total of $46 \mathrm{~min}^{7}$ ) are often too strenuous and/or too long to perform regularly in daily life.

We have been conducting a series of studies to demonstrate that a short period of stair climbing-descending exercise (ST-EX), a convenient method for increasing exercise intensity, is sufficient to reduce postprandial BG levels. ${ }^{11-15}$ ST-EX is easy to perform 
regardless of weather conditions and without the need for any special exercise facility or changing into training clothes. Typically, one set of ST-EX comprises 3-6 min of repetitions of climbing briskly to the second floor followed by walking slowly down to the first floor. The exercise intensity is high during the ascending phase, but it is reduced during the descending phase, thereby increasing the overall intensity without excessive effort. ${ }^{11-14}$ In our recent report, we showed that a single bout of as little as $3 \mathrm{~min}$ of ST-EX performed 60 and 120 min after a meal decreased BG level in people with type 2 diabetes more rapidly than a fully sedentary condition. ${ }^{14}$

We also demonstrated that in people with impaired glucose tolerance (IGT), a 6 min bout of ST-EX starting 90 min after a meal decreased BG level more rapidly than a bout of level walking of the same duration. ${ }^{11}$ However, it is reasonable that ST-EX had a greater hypoglycemic effect than level walking, because the heart rate (HR) during ST-EX was significantly higher than during level walking ( $128 \pm 8$ beats per minute (bpm) vs $113 \pm 9 \mathrm{bpm}$, $\mathrm{p}<0.01$ ), and the estimated average oxygen consumption $\left(\mathrm{VO}_{2}\right)$ of ST-EX was twice that of level walking $(24.8 \pm 2.5$ vs $12.3 \pm 0.7 \mathrm{~mL} / \mathrm{kg} / \mathrm{min}, \mathrm{p}<0.01) .{ }^{11}$

Therefore, the primary purpose of the present study was to confirm the superiority of the acute hypoglycemic effect of ST-EX on postprandial hyperglycemia by comparing it with another type of exercise performed at the same HR as ST-EX. For this comparison, we used constant bicycle exercise (BI-EX) because BI-EX is a popular indoor exercise and its exercise intensity can be manipulated easily and quantitatively. We found that ST-EX ameliorated postprandial BG level more rapidly than BI-EX in people with type 2 diabetes or IGT.

\section{RESEARCH DESIGN AND METHODS}

\section{Subjects}

Seven people with type 2 diabetes $($ male $/$ female $=4 / 3$ ) and seven people with IGT (male $/$ female $=5 / 2$ ) but no macrovascular and microvascular complications, whose physical characteristics and metabolic profiles are shown in (table 1), volunteered for this study. None of the subjects were engaged in any regular endurance or resistance training program at the time of the experiments, had uncontrolled hypertension $(>160 / 100 \mathrm{~mm} \mathrm{Hg}$ ) or an orthopedic disorder that caused physical inactivity, or were taking drugs such as beta-blockers that affect the HR responses to exercise. The subjects were able to ascend and descend stairs without difficulties in their daily life. Five of the subjects with type 2 diabetes were being treated with oral hypoglycemic agents (glimepiride and pioglitazone $(n=1)$; gliclazide $(n=1)$; metformin, pioglitazone and alogliptin $(\mathrm{n}=1)$; metformin, pioglitazone, vildagliptin and acarbose $(n=1)$; or metformin $(n=1))$. The subjects took their medications in the usual way on the experimental days. Written informed consent was obtained from all the participants before the study. The
Table 1 Physical characteristics and metabolic profiles of study subjects

\begin{tabular}{|c|c|c|c|}
\hline & $\begin{array}{l}\text { Type } 2 \\
\text { diabetes }\end{array}$ & IGT & All \\
\hline & $(n=7)$ & $(n=7)$ & $(n=14)$ \\
\hline Age (years) & $70.4 \pm 6.8^{*}$ & $51.3 \pm 3.2$ & $60.9 \pm 11.2$ \\
\hline Height (cm) & $159.4 \pm 10.8$ & $165.0 \pm 4.8$ & $162.2 \pm 8.5$ \\
\hline Body mass (kg) & $58.7 \pm 13.2$ & $66.9 \pm 8.0$ & $62.8 \pm 11.3$ \\
\hline $\begin{array}{l}\text { Body mass index } \\
\left(\mathrm{kg} / \mathrm{m}^{2}\right)\end{array}$ & $22.8 \pm 2.4$ & $24.6 \pm 3.0$ & $23.7 \pm 2.8$ \\
\hline $\mathrm{HbA1c}(\%)$ & $6.8 \pm 0.5^{\star \star}$ & $5.7 \pm 0.2$ & $6.3 \pm 0.6$ \\
\hline $\mathrm{HbA} 1 \mathrm{c}(\mathrm{mmol} / \mathrm{mol})$ & $51 \pm 5^{\star \star}$ & $39 \pm 2$ & $45 \pm 7$ \\
\hline $\begin{array}{l}\text { BG before lunch } \\
(\mathrm{mmol} / \mathrm{L})\end{array}$ & $6.3 \pm 0.4^{\star \star}$ & $5.0 \pm 0.3$ & $5.7 \pm 0.7$ \\
\hline
\end{tabular}

Values are expressed as mean \pm SD.

${ }^{*}$ and ${ }^{* *}$ indicate the presence of significant differences $(p<0.05$ and $p<0.01$, respectively) from IGT.

$B G$, blood glucose; HbA1c, hemoglobin A1c; IGT, impaired glucose tolerance.

study was approved by the Institutional Review Board of Nagoya City University.

\section{Study design}

The experiments were conducted on four separate days over 3 weeks. Intervals of more than 3 days were maintained between the experiments to avoid the metabolic effects of the exercise performed during the previous experiment. It has been shown that a single bout of exercise session increases insulin action and glucose tolerance for more than 24 hours but less than 72 hours. ${ }^{1}$ The subjects were instructed to have a Japanese-style breakfast (rice or bread, and side dishes including broiled fish, rolled omelet and vegetables; $400 \mathrm{kcal}$ ) at home before $07: 30$ and to come to the laboratory by $11: 30$ on each experimental day.

On experimental day 1, the subjects performed the control (REST) trial. The subjects ate a test meal (lunch; $106.5 \mathrm{~g}$ carbohydrate, $18 \mathrm{~g}$ protein, $18 \mathrm{~g}$ fat, $660 \mathrm{kcal})$ between 12:00 and 12:15 and then rested quietly on a chair for $150 \mathrm{~min}$. At $-5,90,105,120$ and $150 \mathrm{~min}$ relative to the start time of lunch, blood samples were collected from a fingertip to determine BG level. At -5 min relative to the start time of lunch, blood samples were drawn from the antecubital vein to determine serum HbAlc level.

After completion of the REST trial, preliminary experiments were conducted to determine the step rate for ST-EX and the work rate for BI-EX that would correspond to an intensity of high-moderate to low-vigorous intensity $(60 \%-65 \%$ of age-predicted HR reserve (HRR) $),{ }^{16}$ and that the subjects were able to perform without a feeling of excessive effort (Borg rating of perceived exertion score of 13-14). ${ }^{17}$ For ST-EX, a straight flight of stairs with 21 steps each $18 \mathrm{~cm}$ high was used. At the stairs, the subjects performed 8-10 repetitions of ST-EX (walking down one flight of stairs followed by climbing up to the 


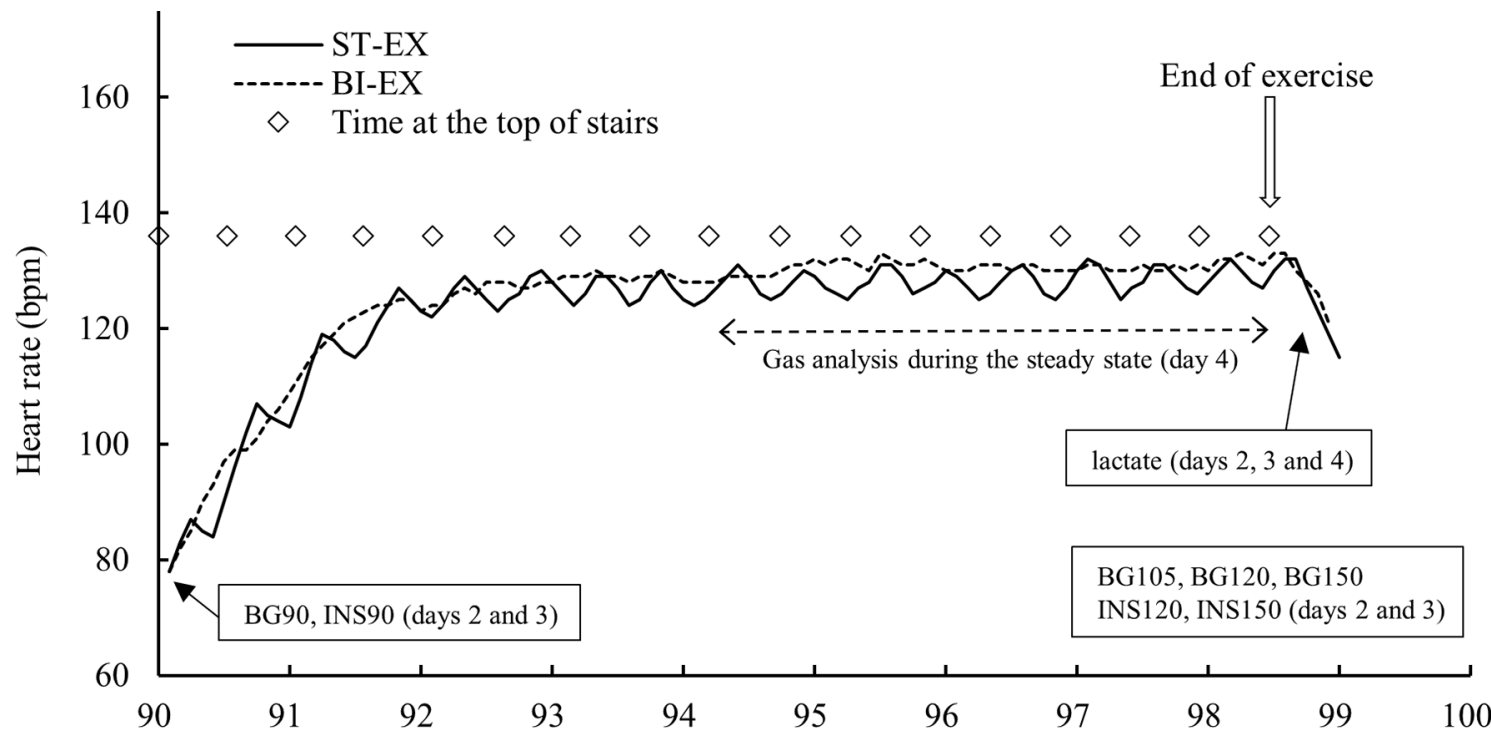

Time after LUNCH (min)

Figure 1 Experimental protocol for days 2, 3 and 4. On days 2 and 3, subjects performed either ST-EX (16 repetitions) or BIEX for the same duration starting $90 \mathrm{~min}$ after lunch. Blood glucose and insulin levels were measured at 90 min (immediately before starting exercise), 105, 120 and $150 \mathrm{~min}$, and 90, 120 and $150 \mathrm{~min}$ after lunch, respectively. On day 4, subjects performed ST-EX and BI-EX in random order with a rest interval of $>50 \mathrm{~min}$. Gas analysis was conducted during the steady state of ST-EX (last 8 repetitions, 4 $\mathrm{min}$ ) and BI-EX (last $4 \mathrm{~min}$ ). Blood lactate was measured $1 \mathrm{~min}$ after exercise on days 2, 3 and 4 . Heart rate was monitored every $5 \mathrm{~s}$ during exercise on days 2, 3 and 4 . BI-EX, bicycle exercise; bpm, beats per minute; ST-EX, stair climbing-descending exercise.

starting point) without rest. At the top and bottom of the stairs, they made a U-turn in 2-3 s. For BI-EX, the subjects performed a pedaling exercise on an isopower-controlled cycle ergometer (232C, Combi, Tokyo, Japan) at their preferred pedaling rate $(50-65$ cycles $/ \mathrm{min})$ for $5-7 \mathrm{~min}$. To avoid excessive fatigue because of delayed cardiac and circulatory responses, the work rate was initially set at $50 \%$ of the individually predetermined work rate for 1 min and thereafter increased to the predetermined work rate. HR was recorded every $5 \mathrm{~s}$ during exercise using an HR monitor (Polar Accurex Plus; Polar Electro, Kempele, Finland).

On experimental day 2 and day 3, the subjects performed either the ST-EX or BI-EX trials in random order (figure 1). We chose the timing for exercise based on previous findings that ST-EX starting at $90 \mathrm{~min}$ after a meal resulted in a rapid and substantial decrease in BG, and did not induce a rebound of BG after exercise. ${ }^{1113}$ For the ST-EX trial, the subjects had lunch between 12:00 and $12: 15$, rested on a chair for $90 \mathrm{~min}$ and then performed 16 repetitions of ST-EX (walking down one flight of stairs followed by climbing up to the starting point) without rest or, if they requested it, a short rest $(<30 \mathrm{~s})$ in standing position at the top of the stairs. They climbed the stairs at an individually predetermined step rate of 80-115 steps/min to the beat of a metronome, and descended at a free step rate, which was lower than the ascending rate for each individual. For the BI-EX trial, the subjects had lunch between 12:00 and 12:15, rested on a chair for $90 \mathrm{~min}$ and then performed the pedaling exercise at an individually predetermined work rate. The duration of BI-EX was the same as that of ST-EX for each subject. We determined the timing for blood sampling based on previous findings that the hypoglycemic effect of ST-EX starting at $90 \mathrm{~min}$ was significant at $105 \mathrm{~min}^{11}{ }^{13}$ and 120 $\min ^{11}$ after a meal. At $-5,90,105,120$ and 150 min relative to the start time of lunch, blood samples were collected from a fingertip to determine the BG level. At 90, 120 and $150 \mathrm{~min}$ relative to the start time of lunch, blood samples were drawn from the antecubital vein to determine serum insulin level. At 1 min after the end of ST-EX or BI-EX, blood samples were collected from a fingertip to determine plasma lactate levels. HR was monitored every 5 s during exercise using Polar Accurex Plus. After the experiment on day 3, the subjects answered the question 'Which exercise (ST-EX or BI-EX) did you feel required less effort?'

On experimental day 4, the subjects performed the ST-EX and BI-EX trials, accompanied by expired gas analysis using the Douglas bag method (figure 1). Three older women with type 2 diabetes were excluded from this investigation to avoid the risk of their falling on the stairs because of interference with their vision by the gas sampling mask. The subjects performed both ST-EX and BI-EX in random order as described above, with a rest interval of at least $50 \mathrm{~min}$ between the exercises. $\mathrm{VO}_{2}$ and respiratory exchange ratio (RER) were determined during the steady state of ST-EX (last 8 repetitions, $\sim 4$ min) and of BI-EX (last 4 min) by a gas analyzer (AR-1, Arco System, Chiba, Japan). HR was monitored every $5 \mathrm{~s}$ 
during exercise. One minute after the end of ST-EX or BI-EX, a blood sample was collected from a fingertip to determine plasma lactate level.

\section{Blood sample analysis}

BG was simultaneously measured in singlicate or duplicate using three different sets of automatic glucose analyzers (Glutest Ace; Arkray, Kyoto, Japan). The average value was calculated. BG0, BG90, BG105, BG120 and BG150 represent $\mathrm{BG}$ at $-5,90,105,120$ and $150 \mathrm{~min}$ from the beginning of lunch, respectively. $\triangle \mathrm{BG} 90-105$, $\triangle B G 90-120$ and $\triangle B G 90-150$ represent the differences in BG between BG90 and BG105, BG90 and BG120, and BG90 and BG150, respectively. Serum insulin level was measured using an immunoassay kit (Architect Insulin, Abbott Japan, Tokyo, Japan). INS90, INS120 and INS150 represent the serum insulin levels 90,120 and $150 \mathrm{~min}$ from the beginning of lunch, respectively. $\triangle \mathrm{INS} 90-120$ and $\triangle \mathrm{INS} 90-150$ represent the difference in serum insulin level between INS90 and INS 120, and between INS90 and INS150, respectively. Plasma lactate level was determined in singlicate using an automated analyzer (Lactate Pro; Arkray). HbA1c level was assayed at Nagoya Clinical Center (Nagoya, Japan) using a latex-agglutination assay.

\section{Statistical analysis}

Data are shown as mean \pm SD except for those in figure 2B. Mann-Whitney $\mathrm{U}$ test was performed to determine whether there were significant differences between type 2 diabetes and IGT (tables 1, 2 and 3). Differences between ST-EX and BI-EX were analyzed by paired analysis using the Wilcoxon signed-rank test (table 2). Comparisons of data between the three trials were performed using the Friedman test (table 3, figure 1). Post-hoc testing of significance between corresponding mean values was performed using the Scheffe method. Significance was set at $\mathrm{p}<0.05$. All statistics were analyzed using SPSS V.18.0 for Windows.

\section{RESULTS}

Physiologic responses to ST-EX and BI-EX

Table 2 shows the physiologic responses to ST-EX and BI-EX in the type 2 diabetes, IGT and combined (ALL) groups measured on experimental day 2 and day 3. HR, $\%$ HRR and exercise duration did not differ significantly between the groups or trials. By contrast, the blood lactate level for ST-EX was significantly lower than that for BI-EX in each group $(\mathrm{p}<0.05)$. Five subjects with type 2 diabetes and four with IGT responded that ST-EX required less effort than BI-EX, and one subject with type 2 diabetes and 2 with IGT answered that both ST-EX and BI-EX required equal effort.

$\mathrm{VO}_{2}$ and RER during the steady state of ST-EX and BI-EX were measured on experimental day 4. There were no differences in the steady-state HR (ST-EX 130.6 $\pm 5.9 \mathrm{bpm}$ vs BI-EX $131.1 \pm 6.7 \mathrm{bpm}$, NS) and \%HRR (ST-EX $63.3 \pm 8.3 \%$ vs BI-EX $63.7 \pm 8.0 \%$,
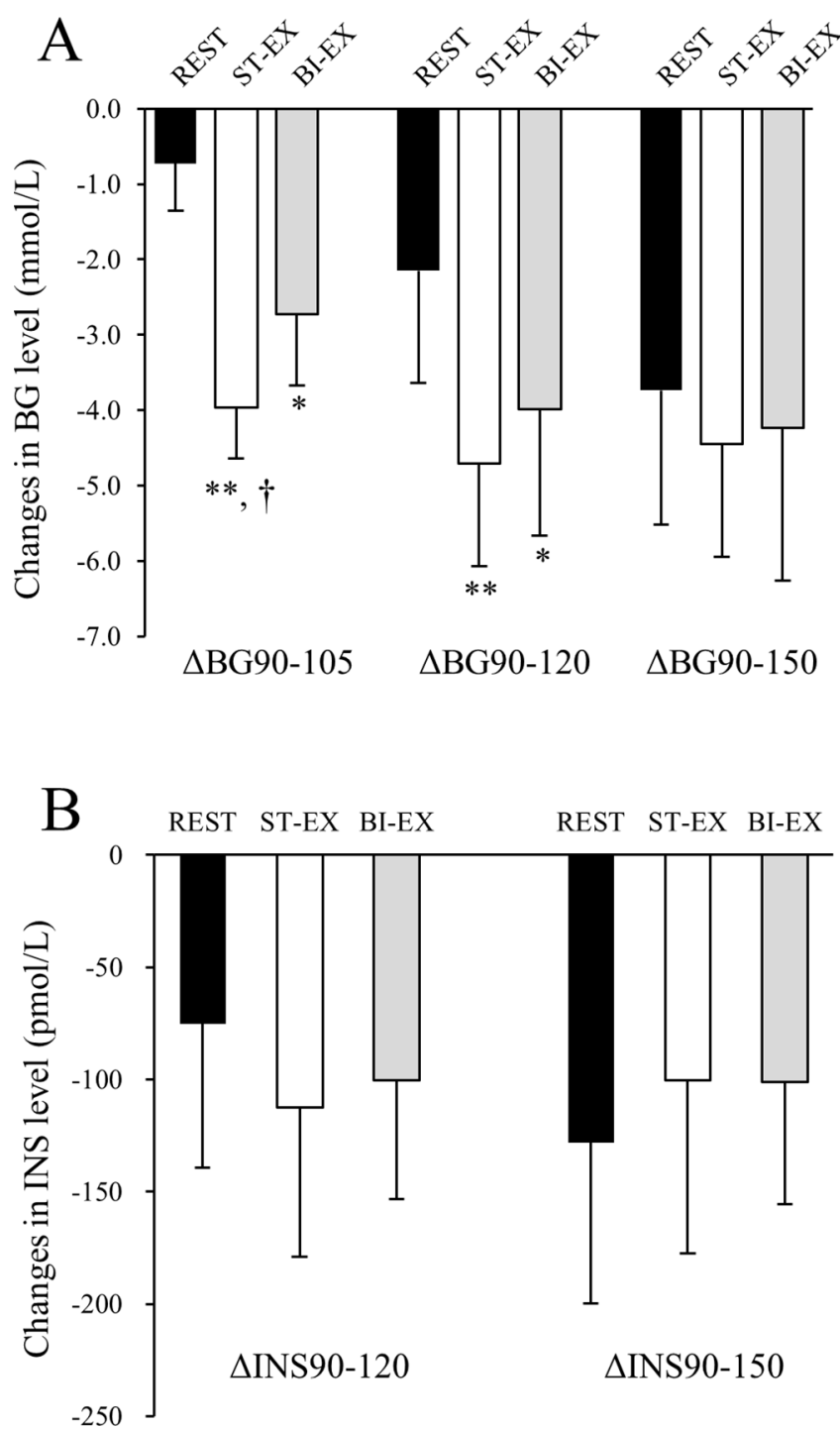

Figure 2 (A) Changes in BG level during REST, ST-EX and BI-EX trials in the combined (ALL) group. ${ }^{*}$ and ${ }^{* *}$ indicate the presence of significant differences $(p<0.05$ and $p<0.01$, respectively) from REST. $\dagger$ indicates the presence of a significant difference $(p<0.05)$ from BI-EX. Values are expressed as mean $\pm S D$. $n=14$. (B) Changes in serum insulin (INS) level during REST, ST-EX and BI-EX trials in the combined group. Values are expressed as mean \pm SE. $n=14$. $B G$, blood glucose; BI-EX, bicycle exercise; INS, serun insulin; ST-EX, stair climbing-descending exercise.

NS). $\mathrm{VO}_{2}$ for ST-EX was significantly higher (by $9 \%$ ) than that for BI-EX (ST-EX $18.7 \pm 1.4 \mathrm{~mL} / \mathrm{kg} / \mathrm{min}$ vs $17.2 \pm 1.3 \mathrm{~mL} / \mathrm{kg} / \mathrm{min}, \mathrm{p}<0.01)$, but RER and blood lactate level for ST-EX were significantly lower than those for BI-EX (RER: ST-EX $0.91 \pm 0.05$ vs BI-EX $0.97 \pm 0.04, \mathrm{p}<0.01$; lactate: ST-EX $3.4 \pm 1.2 \mathrm{mmol} / \mathrm{L}$ vs BI-EX $4.4 \pm 1.0 \mathrm{mmol} / \mathrm{L}, \mathrm{p}<0.01)$.

\section{Changes in BG level after ST-EX and BI-EX}

Table 3 shows the changes in BG level during the REST, ST-EX and BI-EX trials. BG90, that is, BG level immediately before starting ST-EX or BI-EX, for the type 2 
Table 2 Physiologic responses to ST-EX and BI-EX (experimental days 2 and 3 )

\begin{tabular}{|c|c|c|c|}
\hline & $\begin{array}{l}\text { Type } 2 \\
\text { diabetes }\end{array}$ & IGT & All \\
\hline & $(n=7)$ & $(n=7)$ & $(n=14)$ \\
\hline \multicolumn{4}{|l|}{ ST-EX } \\
\hline Average HR (bpm) & $120.3 \pm 4.6$ & $128.1 \pm 8.1$ & $124.2 \pm 7.5$ \\
\hline $\begin{array}{l}\text { Average exercise } \\
\text { intensity (\%HRR) }\end{array}$ & $57.9 \pm 5.5$ & $57.7 \pm 5.5$ & $57.8 \pm 5.3$ \\
\hline $\begin{array}{l}\text { Average steady- } \\
\text { state HR (bpm) }\end{array}$ & $124.4 \pm 3.9$ & $130.7 \pm 7.4$ & $127.6 \pm 6.6$ \\
\hline $\begin{array}{l}\text { Average steady- } \\
\text { state exercise } \\
\text { intensity }(\% \mathrm{HRR})^{\mathrm{b}}\end{array}$ & $62.5 \pm 5.2$ & $60.1 \pm 5.0$ & $61.3 \pm 5.0$ \\
\hline $\begin{array}{l}\text { Steady-state HR } \\
\text { fluctuation }(\mathrm{bpm})^{\mathrm{c}}\end{array}$ & $7.0 \pm 1.6$ & $9.9 \pm 1.9^{\star \star}$ & $8.4 \pm 2.2^{\star *}$ \\
\hline $\begin{array}{l}\text { Blood lactate } \\
(\mathrm{mmol} / \mathrm{L})^{d}\end{array}$ & $3.3 \pm 0.5^{\star}$ & $3.6 \pm 1.3^{*}$ & $3.5 \pm 1.0^{*}$ \\
\hline $\begin{array}{l}\text { Stepping rate } \\
\text { (steps/min) }\end{array}$ & $92.0 \pm 5.4 \dagger$ & $101.9 \pm 7.5$ & $96.9 \pm 8.1$ \\
\hline $\begin{array}{l}\text { Exercise duration } \\
\text { (s) }\end{array}$ & $508 \pm 79$ & $465 \pm 30$ & $486 \pm 62$ \\
\hline \multicolumn{4}{|l|}{ BI-EX } \\
\hline Average HR (bpm) & $122.9 \pm 4.3$ & $127.0 \pm 11.3$ & $124.9 \pm 8.5$ \\
\hline $\begin{array}{l}\text { Average exercise } \\
\text { intensity (\%HRR) }\end{array}$ & $60.7 \pm 4.9$ & $56.6 \pm 9.0$ & $58.6 \pm 7.3$ \\
\hline $\begin{array}{l}\text { Average steady- } \\
\text { state HR (bpm) }{ }^{\text {a }}\end{array}$ & $126.1 \pm 4.0$ & $130.1 \pm 10.5$ & $128.1 \pm 7.9$ \\
\hline $\begin{array}{l}\text { Average steady- } \\
\text { state exercise } \\
\text { intensity }(\% \mathrm{HRR})^{\mathrm{b}}\end{array}$ & $64.1 \pm 5.0$ & $59.6 \pm 8.2$ & $61.9 \pm 6.9$ \\
\hline $\begin{array}{l}\text { Steady-state HR } \\
{\text { fluctuation }(\mathrm{bpm})^{\mathrm{c}}}^{\mathrm{C}}\end{array}$ & $5.6 \pm 1.0$ & $5.3 \pm 1.1$ & $5.4 \pm 1.0$ \\
\hline $\begin{array}{l}\text { Blood lactate } \\
(\mathrm{mmol} / \mathrm{L})^{d}\end{array}$ & $4.3 \pm 1.2$ & $4.7 \pm 1.2$ & $4.5 \pm 1.2$ \\
\hline Work rate $(\mathrm{W})$ & $64.3 \pm 18.8$ & $82.1 \pm 15.5$ & $73.2 \pm 19.0$ \\
\hline $\begin{array}{l}\text { Exercise duration } \\
\text { (s) }\end{array}$ & $506 \pm 78$ & $472 \pm 34$ & $489 \pm 61$ \\
\hline
\end{tabular}

Values are expressed as mean \pm SD.

$\mathrm{a}, \mathrm{b}$ and ${ }^{\mathrm{c}}$ indicate the average $\mathrm{HR}$, average \%HRR and maximal HR minus minimal HR during the last 8 repetitions ( $4 \mathrm{~min})$ of ST$\mathrm{EX}$ or during the last $4 \mathrm{~min}$ of $\mathrm{BI}-\mathrm{EX}$, respectively.

${ }^{d}$ indicates the plasma lactate level at $1 \mathrm{~min}$ after the end of ST-EX or BI-EX.

${ }^{*}$ and ${ }^{* *}$ indicate the presence of significant differences $(p<0.05$ and $p<0.01$, respectively) from BI-EX.

$\dagger$ indicates the presence of a significant difference $(p<0.05)$ from IGT.

BI-EX, bicycle exercise; bpm, beats per minute; HR, heart rate; $\%$ HRR, percentage of age-predicted heart rate reserve; IGT, impaired glucose tolerance; ST-EX, stair climbing-descending exercise.

diabetes group was higher than that for the IGT group $(p<0.05)$, and there were no differences in BG90 between the trials for each subject group. However, $\triangle$ BG90-105
Table 3 Changes in blood glucose (BG) level during REST, ST-EX and BI-EX trials

\begin{tabular}{|c|c|c|c|}
\hline & $\begin{array}{l}\text { Type } 2 \\
\text { diabetes }\end{array}$ & IGT & All \\
\hline & $(n=7)$ & $(n=7)$ & $(n=14)$ \\
\hline \multicolumn{4}{|c|}{ BG90 (mmol/L) } \\
\hline REST & $13.4 \pm 2.5^{\star}$ & $10.8 \pm 0.9$ & $12.1 \pm 2.3$ \\
\hline ST-EX & $12.4 \pm 1.1$ & $11.3 \pm 1.2$ & $11.8 \pm 1.3$ \\
\hline BI-EX & $12.9 \pm 2.0^{\star \star}$ & $11.0 \pm 0.3$ & $11.9 \pm 1.7$ \\
\hline \multicolumn{4}{|c|}{ BG105 (mmol/L) } \\
\hline REST & $12.9 \pm 2.4^{*}$ & $9.9 \pm 0.7$ & $11.4 \pm 2.3$ \\
\hline ST-EX & $8.5 \pm 1.3 \ddagger$ & $7.2 \pm 1.4 \ddagger$ & $7.9 \pm 1.5 \ddagger$ \\
\hline BI-EX & $10.5 \pm 2.1^{*}$ & $7.9 \pm 0.3$ & $9.2 \pm 1.9 \dagger$ \\
\hline \multicolumn{4}{|c|}{ BG120 (mmol/L) } \\
\hline REST & $12.3 \pm 1.9^{\star}$ & $7.7 \pm 1.1$ & $10.0 \pm 2.8$ \\
\hline ST-EX & $8.4 \pm 1.5^{\star \star}, \ddagger$ & $6.0 \pm 1.4 \dagger$ & $7.2 \pm 1.9 \ddagger$ \\
\hline BI-EX & $9.7 \pm 1.9^{\star \star}$ & $6.2 \pm 1.0$ & $8.0 \pm 2.3 \dagger$ \\
\hline \multicolumn{4}{|c|}{ BG150 (mmol/L) } \\
\hline REST & $10.4 \pm 1.8^{\star \star}$ & $6.4 \pm 1.1$ & $8.4 \pm 2.5$ \\
\hline ST-EX & $8.2 \pm 1.5^{\star}$ & $6.6 \pm 1.1$ & $7.4 \pm 1.5$ \\
\hline $\mathrm{BI}-\mathrm{EX}$ & $9.4 \pm 1.7^{\star \star}$ & $6.1 \pm 1.1$ & $7.7 \pm 2.2$ \\
\hline \multicolumn{4}{|c|}{$\Delta \mathrm{BG} 90-105(\mathrm{mmol} / \mathrm{L})$} \\
\hline REST & $-0.5 \pm 0.8$ & $-0.9 \pm 0.4$ & $-0.7 \pm 0.6 \S$ \\
\hline ST-EX & $-3.9 \pm 0.5 \ddagger$ & $-4.1 \pm 0.8 \ddagger$ & $-4.0 \pm 0.7 \neq, \S$ \\
\hline $\mathrm{BI}-\mathrm{EX}$ & $-2.4 \pm 1.2$ & $-3.0 \pm 0.4$ & $-2.7 \pm 0.9 \dagger$ \\
\hline \multicolumn{4}{|c|}{ Net $\triangle B G 90-105(\mathrm{mmol} / \mathrm{L})$} \\
\hline ST-EX & $-3.3 \pm 0.6 \S$ & $-3.1 \pm 0.7 \S$ & $-3.2 \pm 0.79$ \\
\hline BI-EX & $-1.9 \pm 0.8$ & $-2.1 \pm 0.3$ & $-2.0 \pm 0.6$ \\
\hline
\end{tabular}

Values are expressed as mean \pm SD.

BG90, BG105, BG120, BG150: BG level 90, 105, 120 and $150 \mathrm{~min}$ from the beginning of lunch.

$\triangle B G 90-105$ : difference between BG90 and BG105.

Net $\triangle B G 90-105: \triangle B G 90-105$ for ST-EX or BI-EX minus $\triangle B G 90$

105 for REST.

${ }^{*}$ and ${ }^{* *}$ indicate the presence of significant differences $(p<0.05$ and $p<0.01$, respectively) from IGT.

$\dagger$ and $\ddagger$ indicate the presence of significant differences $(p<0.05$ and $p<0.01$, respectively) from REST.

$\S$ and $\|$ indicate the presence of significant differences $(p<0.05$ and $p<0.01$, respectively) from $\mathrm{BI}-\mathrm{EX}$.

BG, blood glucose; BI-EX, bicycle exercise; IGT, impaired glucose tolerance; ST-EX, stair climbing-descending exercise.

for ST-EX was much greater than that for REST in the type 2 diabetes group $(7.5 \times$ higher, $\mathrm{p}<0.05)$, the IGT group (4.3×, $\mathrm{p}<0.01)$ and the combined group (5.5×, $\mathrm{p}<0.01) . \Delta \mathrm{BG} 90-105$ for ST-EX was also $1.5 \times$ greater than that for BI-EX for the combined group $(\mathrm{p}<0.05)$. The net decrease in BG between BG90 and BG105 (net $\triangle \mathrm{BG} 90-105)$ for ST-EX was significantly greater (by $77 \%)$ than that for BI-EX in the type 2 diabetes group $(\mathrm{p}<0.05)$, by $48 \%$ in the IGT group $(\mathrm{p}<0.05)$ and by $62 \%$ in the combined group $(\mathrm{p}<0.01)$. There was no 
difference in net $\Delta$ BG90-105 between type 2 diabetes and IGT groups.

Figure 2A shows the time-course changes in $\triangle \mathrm{BG}$ during the REST, ST-EX and BI-EX trials for the combined group. $\triangle$ BG90-105 for ST-EX was greater than that for either REST $(p<0.01)$ or BI-EX $(p<0.05)$, and $\triangle \mathrm{BG} 90-120$ for ST-EX was greater than that for REST $(p<0.01)$. However, $\triangle$ BG90-120 for ST-EX did not differ from that for BI-EX, and $\triangle B G 90-150$ for ST-EX did not differ from those for BI-EX or REST.

\section{Changes in insulin level after ST-EX and BI-EX}

Figure 2B shows $\Delta$ INS90-120 and $\Delta$ INS90-150 for the combined group. There were no differences in $\triangle \mathrm{INS}$ between the trials for either time period. INS90 also did not differ between the trials $(420 \pm 320,310 \pm 306$ and $322 \pm 259 \mathrm{mmol} / \mathrm{L}$ for REST, ST-EX and BI-EX trials, respectively).

\section{DISCUSSION}

The novel finding of the present study is that an approximately 8 min bout of ST-EX starting 90 min after a meal decreased postprandial BG level more rapidly than REST and BI-EX performed at the same HR in subjects with IGT and type 2 diabetes, although the serum insulin levels did not differ between the three groups. It has been shown that changes in insulin level during exercise are related to the intensity and duration of the exercise. For example, Larsen $e t a l^{18}$ showed that moderate-intensity to high-intensity BI-EX for $45 \mathrm{~min}$ during the ascending phase of BG after a meal blunted the postprandial increase in insulin level in people with type 2 diabetes. Insulin secretion was decreased in response to the reduction in BG level induced by exercise and by the induction of a counter-regulatory response during exercise, including increased sympathetic nervous activity. ${ }^{19}$ Our data suggest that neither ST-EX nor BI-EX elicited a strong counter-regulatory effect, and that insulin secretion was reduced as a secondary response to the decreased BG levels.

It is well established that contraction rapidly activates glucose uptake in skeletal muscle by inducing translocation of glucose transporter 4 (GLUT4) to the cell surface (contraction-stimulated glucose uptake). ${ }^{20}{ }^{21}$ It has been shown in humans that glucose uptake is increased within $5 \mathrm{~min},{ }^{22}{ }^{23}$ and reaches a near-maximum within $10 \mathrm{~min}$ after the start of exercise. ${ }^{24}$ In rat skeletal muscle incubated in vitro a single $10 \mathrm{~s}$ contraction by electrical stimulation (ES) was sufficient to initiate increased glucose uptake, and maximal glucose uptake was achieved after 10 contractions (once per minute).$^{25}$ This increase was maintained for at least $10 \mathrm{~min}$ after stimulation and had decreased by only $50 \%$ after 60 min. ${ }^{25}$ In another study of rat skeletal muscle, $34 \%$ of the initial level of glucose uptake was maintained 3 hours after exercise. ${ }^{26}$ Importantly, contraction-stimulated glucose uptake and GLUT4 translocation in humans remain at normal levels in insulin-resistant conditions such as type 2 diabetes and obesity. ${ }^{27-29}$ These exercise-evoked mechanisms have been widely applied for acute amelioration of high BG, particularly postprandial hyperglycemia.

Our results appeared to show a discrepancy between $\mathrm{VO}_{2}$ and blood lactate/RER values (see 'Physiologic responses to ST-EX and BI-EX'). Although the results for $\mathrm{VO}_{2}$ indicate that the exercise intensity for ST-EX was higher than that for BI-EX, blood lactate/RER results suggest that the exercise intensity was lower for ST-EX than for BI-EX. Moreover, 9 of 14 (64\%) subjects responded that ST-EX required less effort than BI-EX did. The rate of carbohydrate utilization for both aerobic and anaerobic energy production increases as the work intensity increases. ${ }^{23}{ }^{30}$ In the present study, the average power during the ascending phase of ST-EX for the combined group was estimated to be $\sim 180 \mathrm{~W}(62.5(\mathrm{~kg}) \times 9.8(\mathrm{~m} /$ $\left.\mathrm{s}^{2}\right) \times 0.18(\mathrm{~m}) \times 96.9 / 60($ steps $\left./ \mathrm{s})\right)$. When an individual ascends stairs continuously at this work rate, their estimated rate of $\mathrm{VO}_{2}$ is $\sim 39 \mathrm{~mL} / \mathrm{kg} / \mathrm{min}(2.03 \times 0.18(\mathrm{~m} /$ step) $\times 96.9($ steps $/ \mathrm{min})+3.7) .{ }^{12}$ We did not determine the aerobic capacity of the study subjects, but considering their age and their lack of current participation in exercise programs, it is highly likely that a large part of their energy production during the ascending phase of ST-EX was dependent on anaerobic glycolysis. However, our ST-EX protocol consisted of a short ascending phase ( 13 s) followed by a long descending phase with U-turns on a flat floor $(\sim 18 \mathrm{~s})$. We consider that for most of the study subjects, this alternative exercise regimen of ST-EX decreased the blood lactate/RER values below the levels generated by BI-EX and alleviated the perceived exertion while maintaining $\mathrm{VO}_{2}$ at higher levels than with BI-EX. In this regard, it should be noted that a light-intensity or moderate-intensity exercise subsequent to a high-intensity exercise has an 'active recovery' effect, which clears blood lactate faster than 'passive recovery'. 3132

Because the rate of glucose uptake is closely correlated with exercise intensity represented by $\mathrm{VO}_{2}$ during exercise, ${ }^{22-24}$ to some extent, the small $(9 \%)$ but significant difference in $\mathrm{VO}_{2}$ between ST-EX and BI-EX may contribute to the significant difference in the reduction in BG produced by ST-EX and BI-EX. However, different modes of exercise often induce different physiologic and metabolic responses even at the same $\mathrm{VO}_{2}$. We previously compared the effect of ES of lower limb muscles with that of voluntary contractions (VC) at the same $\mathrm{VO}_{2}$ using a glucose clamp procedure. ${ }^{33}$ In healthy subjects lying in the supine position, both lower limb muscles were stimulated through surface electrodes to contract for $20 \mathrm{~min}(20 \mathrm{~Hz}$ stimulation frequency with $1 \mathrm{~s}$ on-off duty cycle). On a different day, the subjects also performed supine VC for 20 min using a cycle ergometer. Despite the identical elevation of $\mathrm{VO}_{2}$ from baseline (ES $7.3 \pm 0.3 \mathrm{~mL} / \mathrm{kg} / \mathrm{min}$ vs VC $7.5 \pm 0.3 \mathrm{~mL} / \mathrm{kg} /$ min, NS), ES increased the blood lactate concentration from $1.2 \pm 0.1$ to $3.2 \pm 0.3 \mathrm{mmol} / \mathrm{L}(\mathrm{p}<0.01)$ after the initiation of ES, whereas VC caused no such increase. ES also 
elevated RER more strongly than did VC (ES $0.99 \pm 0.03$ vs VC $0.83 \pm 0.03, \mathrm{p}<0.01)$. Furthermore, the increase in glucose disposal rate disappeared within $50 \mathrm{~min}$ after the cessation of VC, while it was maintained for at least 90 min after the cessation of ES. These observations clearly indicate that $\mathrm{VO}_{2}$ is not the 'gold standard' for adjusting the exercise intensity of different exercise types, particularly when examining glucose uptake and glycolysis in contracting skeletal muscles. In the present study, we used $\mathrm{HR}$, instead of $\mathrm{VO}_{2}$, to adjust the exercise intensity of ST-EX and BI-EX, because it is one of the most widely used modalities for monitoring exercise intensity in clinical practice. ${ }^{16}$

We note that the body mass index (BMI) of Japanese people with type 2 diabetes is lower than that of Westerners. For instance, the average BMI of participants in the UK Prospective Diabetes Study was $29.4 \mathrm{~kg} / \mathrm{m}^{2}(\mathrm{n}=2015)$, while that of the participants in the Japan Diabetes Complication Study was only $23.1 \mathrm{~kg} / \mathrm{m}^{2}(\mathrm{n}=2205) .{ }^{34}$ The BMI of the subjects of this study was comparable with the Japanese value (table 1). Because the maximal cardiorespiratory fitness of obese individuals with type 2 diabetes is often as low as 7-9 metabolic equivalents (METs) ${ }^{35} 36$ there may be concern that ST-EX is too strenuous for obese patients and is not practically applicable to such subjects. In this regard, we previously measured $\mathrm{VO}_{2}$ in Japanese adults who performed ST-EX with or without a weight jacket to adjust each subject's BMI to 25, 30 and $35 .^{12}$ These experiments clearly demonstrated that $\mathrm{VO}_{2}$ values were almost equal regardless of the change in BMI (18.8-20.2 $\mathrm{mL} / \mathrm{kg} / \mathrm{min}$ (5.4-5.8 METs at $90 \mathrm{steps} / \mathrm{min})$. We consider that the exercise intensity of ST-EX per se is within a well-tolerated level not only in normal-weight patients but in most obese patients.

The clinical significance of the differences in the decrease in postprandial BG after ST-EX and after BI-EX needs to be clarified in a future study. Continuous glucose monitoring may be helpful to analyze precisely the $\mathrm{BG}$ responses after both types of exercises. We recently conducted a pilot study to explore the practical relevance of the differences in the decrease in postprandial BG in people with type 2 diabetes with and without ST-EX. ${ }^{15}$ Seven men with type 2 diabetes who took oral hypoglycemic agents (age 68.0 03.7 years) performed two sets of ST-EX 60 and 120 min after each meal for the first 2 weeks, but not for the following 2 weeks. Each set of exercises, which were performed at home, comprised 3 min of continuous repetition of climbing briskly to the second floor at a rate of 80-110 steps/min followed by slow walking down to the first floor at a free step rate. A rest period of 1-2 min was allowed between each set. We found that the serum 1,5-anhydroglucitol level significantly increased by $11.5 \%$ from baseline at the end of the 2-week exercise period $(\mathrm{p}<0.05)$, and returned to the baseline value at the end of the following 2-week period. These results indicate that a home-based postprandial ST-EX program may improve the overall glycemic control in sedentary people with type 2 diabetes.
In summary, an $8 \mathrm{~min}$ bout of ST-EX starting $90 \mathrm{~min}$ after a meal and comprising 16 repetitions of walking down one flight of stairs followed by brisk climbing up to the starting point has an acute hypoglycemic effect in people with IGT and type 2 diabetes. Compared with BI-EX performed at the same HR, ST-EX more rapidly ameliorated postprandial hyperglycemia, although the blood lactate level and RER were significantly lower and $\mathrm{VO}_{2}$ was higher for ST-EX than BI-EX. Based on these observations and our previous reports, ${ }^{11-15}$ we propose that a short bout of ST-EX is a potentially useful method to improve glucose excursion after meals. The exercise regimen of ST-EX (eg, step rate, number of repetitions, duration of rest between the repetitions and so on) should be individually adjusted according to clinical condition.

Acknowledgements The authors gratefully acknowledge the helpful advice of Dr. Sung Chul Lee. The authors also express their sincere gratitude to the study participants for their time and effort.

Contributors TT conceived, designed and performed the experiments. TT and TH analyzed the data and wrote the manuscript. Both authors approved the final version of the manuscript. Both authors are the guarantors of this work.

Funding This work was supported by Grants-in-Aid for Research from Nagoya City University; JSPS KAKENHI (15K01711) and research grants from the Japanese Council for Science, Technology and Innovation, SIP (project ID: 14533567) (funding agency: Bio-oriented Technology Research Advancement Institution, NARO); Ministry of Agriculture, Forestry and Fisheries, Integration Research for Agriculture and Interdisciplinary Fields (project ID: 14532022) (funding agency: Bio-oriented Technology Research Advancement Institution, NAR0); and Vascular Disease Research Foundation.

\section{Competing interests None declared.}

Ethics approval The Institutional Review Board of Nagoya City University. Provenance and peer review Not commissioned; externally peer reviewed. Data sharing statement This manuscript includes all the data available.

Open Access This is an Open Access article distributed in accordance with the Creative Commons Attribution Non Commercial (CC BY-NC 4.0) license, which permits others to distribute, remix, adapt, build upon this work non-commercially, and license their derivative works on different terms, provided the original work is properly cited and the use is non-commercial. See: http://creativecommons.org/ licenses/by-nc/4.0/

(C) Article author(s) (or their employer(s) unless otherwise stated in the text of the article) 2017. All rights reserved. No commercial use is permitted unless otherwise expressly granted.

\section{REFERENCES}

1. Colberg SR, Albright AL, Blissmer BJ, et al. Exercise and type 2 diabetes: American College of Sports Medicine and the American Diabetes Association: joint position statement. Exercise and type 2 diabetes. Med Sci Sports Exerc 2010;42:2282-303.

2. Tominaga $\mathrm{M}$, Eguchi $\mathrm{H}$, Manaka $\mathrm{H}$, et al. Impaired glucose tolerance is a risk factor for cardiovascular disease, but not impaired fasting glucose. The Funagata Diabetes Study. Diabetes Care 1999;22:920-4

3. DECODE Study Group, the European Diabetes Epidemiology Group. Glucose tolerance and cardiovascular mortality: comparison of fasting and 2-hour diagnostic criteria. Arch Intern Med 2001;161:397-405.

4. Nakagami T. DECODA Study Group. Hyperglycaemia and mortality from all causes and from cardiovascular disease in five populations of Asian origin. Diabetologia 2004;47:385-94.

5. American Diabetes Association. 6. Glycemic Targets. Diabetes Care 2017;40:S48-S56.

6. Bonora E, Calcaterra F, Lombardi S, et al. Plasma glucose levels throughout the day and $\mathrm{HbA}(1 \mathrm{c})$ interrelationships in type 2 diabetes: 
implications for treatment and monitoring of metabolic control. Diabetes Care 2001;24:2023-9.

7. Larsen JJ, Dela F, Madsbad S, et al. The effect of intense exercise on postprandial glucose homeostasis in type II diabetic patients. Diabetologia 1999;42:1282-92.

8. Gillen JB, Little JP, Punthakee Z, et al. Acute high-intensity interval exercise reduces the postprandial glucose response and prevalence of hyperglycaemia in patients with type 2 diabetes. Diabetes Obes Metab 2012;14:575-7.

9. Francois ME, Baldi JC, Manning PJ, et al. 'Exercise snacks' before meals: a novel strategy to improve glycaemic control in individuals with insulin resistance. Diabetologia 2014;57:1437-45.

10. Karstoft K, Christensen CS, Pedersen BK, et al. The acute effects of interval- Vs continuous-walking exercise on glycemic control in subjects with type 2 diabetes: a crossover, controlled study. J Clin Endocrinol Metab 2014;99:3334-42.

11. Takaishi T, Imaeda K, Tanaka T, et al. A short bout of stair climbingdescending exercise attenuates postprandial hyperglycemia in middle-aged males with impaired glucose tolerance. Appl Physiol Nutr Metab 2012;37:193-6.

12. Takaishi $T$, Ishihara $K$, Shima $N$, et al. Health promotion with stair exercise. J Phys Fit Sports Med 2014;3:173-9.

13. Takaishi T, Hayashi T. Stair climbing/descending exerciseimmediate effect against postprandial hyperglycemia in older people with type 2 diabetes mellitus. Ann Sports Med Res 2015;2:1023.

14. Honda $\mathrm{H}$, Igaki $\mathrm{M}$, Hatanaka $\mathrm{Y}$, et al. Stair climbing/descending exercise for a short time decreases blood glucose levels after a meal in people with type 2 diabetes. BMJ Open Diabetes Res Care 2016:4:e000232.

15. Honda $\mathrm{H}$, Igaki $\mathrm{M}$, Hatanaka $\mathrm{Y}$, et al. Repeated 3-minute stair climbing-descending exercise after a meal over 2 weeks increases serum 1,5-anhydroglucitol levels in people with type 2 diabetes. $J$ Phys Ther Sci 2017;29:75-8.

16. Garber CE, Blissmer B, Deschenes MR, et al. American College of Sports Medicine position stand. Quantity and quality of exercise for developing and maintaining cardiorespiratory, musculoskeletal, and neuromotor fitness in apparently healthy adults: guidance for prescribing exercise. Med Sci Sports Exerc 2011;43:1334-59.

17. Borg G. Perceived exertion as an indicator of somatic stress. Scand J Rehabil Med 1970;2:92-8.

18. Larsen JJ, Dela F, Kjaer M, et al. The effect of moderate exercise on postprandial glucose homeostasis in NIDDM patients. Diabetologia 1997;40:447-53.

19. Kjaer M, Hollenbeck CB, Frey-Hewitt B, et al. Glucoregulation and hormonal responses to maximal exercise in non-insulin-dependent diabetes. J Appl Physiol 1990;68:2067-74.

20. Hayashi T, Wojtaszewski JF, Goodyear LJ. Exercise regulation of glucose transport in skeletal muscle. Am J Physiol 1997;273(6 Pt 1):E1039-51
21. Jessen N, Goodyear LJ. Contraction signaling to glucose transport in skeletal muscle. J Appl Physiol 2005;99:330-7.

22. Wahren J, Felig P, Ahlborg G, et al. Glucose metabolism during leg exercise in man. J Clin Invest 1971;50:2715-25.

23. Romijn JA, Coyle EF, Sidossis LS, et al. Regulation of endogenous fat and carbohydrate metabolism in relation to exercise intensity and duration. Am J Physiol 1993;265(3 Pt 1):E380-91.

24. Felig P, Wahren J. Fuel homeostasis in exercise. N Engl $\mathrm{J}$ Med 1975;293:1078-84.

25. Musi N, Hayashi T, Fujii N, et al. AMP-activated protein kinase activity and glucose uptake in rat skeletal muscle. Am J Physiol Endocrinol Metab 2001;280:E677-84.

26. Wallberg-Henriksson H, Constable SH, Young DA, et al. Glucose transport into rat skeletal muscle: interaction between exercise and insulin. J Appl Physiol 1988;65:909-13.

27. Minuk HL, Vranic M, Marliss EB, et al. Glucoregulatory and metabolic response to exercise in obese noninsulin-dependent diabetes. Am J Physiol 1981;240:E458-64.

28. Martin IK, Katz A, Wahren J. Splanchnic and muscle metabolism during exercise in NIDDM patients. Am J Physiol 1995;269(3 Pt 1):E583-90.

29. Kennedy JW, Hirshman MF, Gervino EV, et al. Acute exercise induces GLUT4 translocation in skeletal muscle of normal human subjects and subjects with type 2 diabetes. Diabetes 1999;48:1192-7.

30. van Loon LJ, Greenhaff PL, Constantin-Teodosiu D, et al. The effects of increasing exercise intensity on muscle fuel utilisation in humans. $J$ Physiol 2001;536(Pt 1):295-304.

31. Martin NA, Zoeller RF, Robertson RJ, et al. The comparative effects of sports massage, active recovery, and rest in promoting blood lactate clearance after supramaximal leg exercise. J Athl Train 1998;33:30-5.

32. Menzies P, Menzies C, McIntyre L, et al. Blood lactate clearance during active recovery after an intense running bout depends on the intensity of the active recovery. J Sports Sci 2010;28:975-82.

33. Hamada T, Hayashi T, Kimura T, et al. Electrical stimulation of human lower extremities enhances energy consumption, carbohydrate oxidation, and whole body glucose uptake. J Appl Physiol 2004;96:911-6.

34. Sone $\mathrm{H}$, Ito $\mathrm{H}$, Ohashi $\mathrm{Y}$, et al. Obesity and type 2 diabetes in Japanese patients. Lancet 2003;361:85.

35. Wing RR, Jakicic J, Neiberg R, et al. Fitness, fatness, and cardiovascular risk factors in type 2 diabetes: look ahead study. Med Sci Sports Exerc 2007:39:2107-16.

36. Zaccardi F, O'Donovan G, Webb DR, et al. Cardiorespiratory fitness and risk of type 2 diabetes mellitus: a 23-year cohort study and a meta-analysis of prospective studies. Atherosclerosis 2015;243:131-7. 